\title{
Uso indiscriminado de pesticidas y ausencia de control sanitario para el mercado interno en Perú
}

\author{
Jaime Delgado-Zegarra1 , Aldo Alvarez-Risco² y Jaime A. Yáñez ${ }^{3,4}$
}

Forma de citar Delgado-Zegarra J, Alvarez-Risco A, Yáñez JA. Uso indiscriminado de pesticidas y ausencia de control sanitario para el mercado interno en Perú. Rev Panam Salud Publica. 2018;42:e3. https://doi. org /10.26633/RPSP.2018.3

RESUMEN Notificar el nivel de contaminación de los alimentos de origen animal y vegetal monitorizados por la autoridad sanitaria en Perú, que realiza el Servicio Nacional de Sanidad Agraria (SENASA), para poder informar al consumidor y a las autoridades nacionales de la situación del control sanitario del mercado interno en el país.

La información se ha obtenido de los informes incluidos en la página web del SENASA. Los datos considerados corresponden a los de los informes de evaluación de todos los tipos de alimentos de origen vegetal y animal analizados en el período comprendido entre 2011 y 2015.

Para este período, los resultados muestran que 202 muestras de animales y vegetales incumplen las normas (son no conformes). El porcentaje de muestras de alimentos de origen animal no conformes fue $12,68 \%$ y el correspondiente a alimentos de origen vegetal, 24,87\%. En el periodo estudiado, se observó un aumento de 30,73\% de las muestras no conformes, que incluso ha alcanzado $50 \%$.

Los niveles de contaminación de los alimentos de origen animal y vegetal monitorizados por el SENASA son preocupantes. Por ello, se recomienda iniciar acciones concretas para afrontar esta situación, confiando en que estas acciones sean priorizadas y planificadas con la participación de todos los actores del sistema agrícola de Perú a fin de introducir los cambios regulatorios necesarios y establecer indicadores claros, medibles y alcanzables de monitorización y control para proteger la salud de la población.

Palabras clave Alimentos; contaminantes; contaminación ambiental; regulación gubernamental; Perú.

La autoridad encargada de controlar el uso de plaguicidas en los alimentos en Perú es el Servicio Nacional de Sanidad Agraria (SENASA), que, por mandato legal, ha estado monitorizando los

\footnotetext{
Universidad de San Martin de Porres, Facultad de Ciencias Administrativas y Recursos Humanos, Instituto de Consumo, Lima, Perú. La correspondencia se debe dirigir a Jaime Delgado. Correo electrónico: jdelgadoz@usmp.pe

2 Universidad de San Martin de Porres, Facultad de Ciencias Administrativas y Recursos Humanos, Instituto de Investigación, Lima, Perú.
}

alimentos para detectar la presencia de pesticidas no permitidos. Estas actividades están amparadas en la Ley de Inocuidad de Alimentos, DL 1062 (1) mediante sus reglamentos DS

\footnotetext{
Departamento de Investigación, Desarrollo e Innovación, Indetec Corp SAC, Lima, Perú.

4 YARI International Group, LLC, New Brunswick, New Jersey, Estados Unidos de América.
}

No. 034-2008-AG (2) y DS No. 04-2011AG (3).

El Artículo No. 32 del último reglamento (3) dispone que "SENASA establecerá el Programa Nacional de Monitoreo de Contaminantes que afecta la seguridad de los alimentos y piensos primarios agropecuarios que puedan poner en peligro la salud de las personas; este Programa consistirá en planes anuales que involucren el ámbito geográfico, el tipo de alimento, el número de muestras a analizar, así como los procedimientos a seguir".

Este es un artículo de acceso abierto distribuido bajo los términos de la licencia Creative Commons Attribution-NonCommercial-NoDerivs 3.0 IGO, que permite su uso, distribución y reproducción en cualquier medio, siempre que el trabajo original se cite de la manera adecuada. No se permiten modificaciones a los artículos ni su uso comercial. Al reproducir un artículo no debe haber ningún indicio de que la OPS o el artículo avalan a una organización o un producto específico. El uso del logo de la OPS no está permitido. Esta leyenda debe conservarse, junto con la URL original del artículo. 
En cumplimiento de este mandato, se publicaron la Resolución Jefatural No. 141-2011-AG-SENASA y la Resolución Directoral No.107-2011-AG-SENASADIAIA (4), que aprobó el Programa Nacional de Monitoreo de Contaminantes en Alimentos Primarios Agrícolas y Piensos.

Las pruebas científicas actuales disponibles en la bibliografía describen la presencia de antibióticos en los agroecosistemas, como, por ejemplo, las que aportan Williams-Nguyen, et al (5) y Aga, et al (6) y el informe especial de la Organización Mundial de la Salud (OMS), en las cuales se indica que las resistencias bacterianas a los antibióticos ya son una realidad a escala mundial (7).

Por estas razones, el objetivo de este estudio es dar a conocer el nivel de contaminación de los alimentos de origen animal y vegetal que son monitorizados por la autoridad sanitaria en Perú, para poder informar a los consumidores y a las autoridades de la situación del control sanitario del mercado interno en el país.

\section{MATERIALES Y MÉTODOS}

Se realizó un análisis detallado de los informes disponibles en la página web del SENASA (www.senasa.gob.pe). Los informes que se usaron en la presente investigación son aquellos vinculados con el Monitoreo de Contaminantes Químicos en Alimentos Agropecuarios Primarios. El periodo que cubrió este estudio fue aquel sobre el cual se disponía de información en la página web del SENASA: 2011-2015 (8-12). De estos informes, se extrajo información sobre los tipos de alimentos, tanto de origen animal como de origen vegetal, el tipo de contaminación detectado, el número de muestras analizadas $\mathrm{y}$, de ellas, el número de muestras conformes y no conformes y el porcentaje de muestras que correspondía a cada categoría (conformes y no conformes respecto al total de muestras).

Para entender los informes del SENASA han de conocerse algunos conceptos. Primero, los límites máximos de residuos (LMR) son la cantidad máxima de estos residuos que pueden contener los alimentos. Están contenidos en el Codex Alimentarius y en Perú se han adoptado e incorporado en la norma nacional. Segundo, el de muestra no conforme, que se define como aquella muestra que contenga cualquier ingrediente activo de residuo de pesticida químico de uso agrícola o medicamento veterinario que se emplee en usos no aprobados por la Autoridad Nacional o que contenga una cantidad de residuo de la sustancia activa superior al LMR establecido por el Codex Alimentarius. Tercero, el de contaminación en alimentos de origen vegetal y animal, que se entiende como la presencia de medicamentos veterinarios, plaguicidas no registrados, residuos químicos, contaminantes microbiológicos, como bacterias o micotoxinas y metales pesados no autorizados.

\section{RESULTADOS}

Los informes del periodo comprendido entre 2011 y 2015 emitidos por el SENASA muestran los resultados de la detección de la presencia de contaminantes por agentes químicos en alimentos agrícolas primarios (8-12). Los resultados del cumplimiento y de las muestras no conformes para este periodo se detallan en el cuadro 1. En él se aprecia la disminución de 30,73\% de las muestras conformes, lo que pone de relieve que hasta $50 \%$ de las muestras no son conformes.

El informe No. 0032-2015-MINAGRISENASA-DIAIA-JCARRASCO, elaborado por el SENASA, detalla los productos agrícolas que han sido rechazados por la FDA para su importación en los Estados Unidos de América en 2014 y 2015. En total, se contabilizan 69 documentos en los cuales se notifica el rechazo de quinua blanca, semilla de quinua, paprika entero, tangelo, lechuga de cabeza y arveja, entre otros (13). A continuación, se comentan distintos aspectos críticos de los alimentos analizados a partir del informe de 2013 (10).

Contaminación de factores alimentarios. El principal aspecto que explica la contaminación es el uso no adecuado de insumos químicos en el trabajo agrícola relacionado con la producción y el procesamiento primario, además del asesoramiento, la orientación y la supervisión deficientes en el uso de estos productos por parte de los fabricantes o de las autoridades del sector.

Los productos vegetales seleccionados fueron aceituna, alcachofa, plátano, café, cebolla, espárrago, limón, mandarina, mango, naranja, nueces de Brasil, pallar, aguacate, pimentón, tomate y uva. Los alimentos de origen animal fueron carnes de pollo, ovejas, cerdo, camélidos sudamericanos, cobayos, cabras, pavos, miel y leche.

La monitorización realizada ha demostrado que algunas muestras contenían sustancias prohibidas en Perú, como productos para uso veterinario (cloranfenicol y nitrofuranos) y sustancias que exceden los límites máximos establecidos en el Codex Alimentarius. Los alimentos de origen animal más contaminados con residuos químicos fueron la carne de pollo y la carne de vacuno. El mayor nivel de contaminantes microbiológicos se encontró en la carne bovina, la caprina, la ovina y en el pollo y Escherichia coli y Staphylococcus aureus fueron las bacterias encontradas con mayor frecuencia. El arsénico y el cadmio fueron los metales pesados que se encontraron principalmente en los alimentos para animales controlados.

En las siguientes secciones se detalla el tipo de contaminación que se ha encontrado en los alimentos con mayor incidencia de contaminación.

Tomate con agroquímicos. El tomate es uno de los alimentos más consumidos a nivel mundial, incluido el Perú (15-16), y es preocupante que sea uno de los productos más desaprobados por la presencia de pesticidas (10), como indican los informes de 2012 a 2014, en los cuales los porcentajes de informes no conformes fueron, respectivamente, 74, 55 y 73\%. (En los informes de 2011 y 2015 no se notificaron estos porcentajes.)

Paprika con micotoxinas. En el caso de paprika, un producto emblemático para la exportación, llama la atención la alta prevalencia de la presencia de micotoxinas ocratoxina A y aflatoxina B1 en $33,3 \%$ de las muestras, que son nocivas para la salud humana y se consideran altamente tóxicas e incluso cancerígenas $(10,17,18)$.

Antibióticos en la carne. En la carne de cerdo se detectaron 2 muestras de un total de 5 en la provincia de Cajamarca con niveles de oxitetraciclina por encima de los niveles permitidos. Estas muestras procedían de los Mercados Centrales y el Mercado Baños del Inca (10). En la provincia de Tacna, todas las muestras analizadas (7 muestras de un total de 7) fueron no conformes, ya que 5 muestras tuvieron la presencia de metabolitos de furazolidona y las otras 2 , de metabolitos de furaltadona. La presencia de furazolidona en los alimentos en la provincia de Lambayeque y La Parada en la ciudad de Lima se detectó en 12,5 y $20 \%$ de las muestras analizadas en Lambayeque y Lima, respectivamente (10). 
CUADRO 1. Muestras conformes y no conformes de alimentos de origen animal y vegetal según los Informes del Monitoreo de Contaminantes Químicos en Alimentos Agropecuarios Primarios de 2011 a 2015 del Servicio Nacional de Sanidad Agraria, Lima, Perú

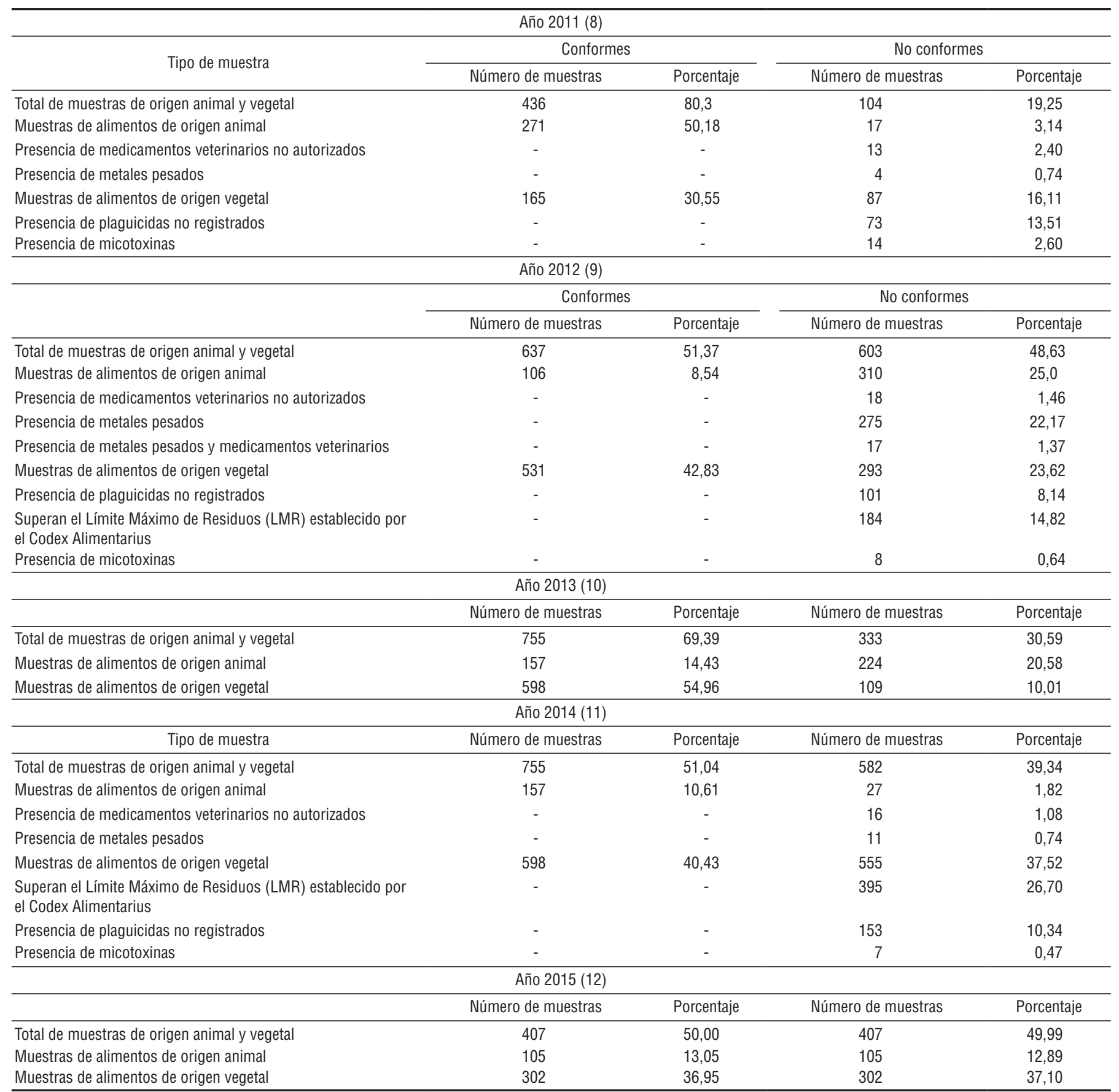

Fuente: elaboración propia a partir de los resultados obtenidos de los Informes del Monitoreo de Contaminantes Químicos en Alimentos Agropecuarios Primarios de 2011 a 2015 realizados por el Servicio Nacional de Sabidad Agraria de Perú.

Mandarinas con plaguicidas no autorizados. En las mandarinas, que son una fruta muy consumida en Perú, la presencia de productos químicos no autorizados también fue muy elevada en virtud de los niveles establecidos en el Decreto Supremo No. 001-2015 del
Ministerio de Agricultura y Riego (MINAGRI) (20). Las sustancias detectadas con mayor frecuencia fueron hexitiazox, acetamiprid, piridabeno, tiabendazol y fenil fenol. De las 41 muestras analizadas, $20(48,8 \%)$ fueron no conformes (10).
Naranja con plaguicidas no autorizados. Una situación similar se observó con las naranjas, tan comúnmente consumidas en el país, ya que del total de muestras analizadas, 11 de ellas $(22,4 \%)$ no cumplían la normativa. La sustancias detectadas fueron, entre otras, miclobutanil, 
etoprofos, aldicarb sulfóxido y tebuconazol y la ciudad de Lima y las provincias de Piura, Ica y Libertad fueron los lugares donde más se utilizaban estas sustancias (10).

Uva con plaguicidas no autorizados. En el caso de las uvas, los resultados también fueron muy similares a los anteriores, ya que de las 45 muestras analizadas 31 de ellas $(68,9 \%)$ incumplían la normativa por contener carbendazim, difenoconazol y otros. En las provincias de San Martín, Tacna y Arequipa se detectaron los porcentajes más altos de uvas con estas sustancias (10).

Arsénico. Según el informe del SENASA de 2011, de las 288 muestras de carne animal analizadas (aves de corral, bovinos, ovinos y caprinos) se detectó la presencia de arsénico en 131 de ellas, es decir en $45,4 \%$, si bien ninguno de los valores detectados sobrepasaba las dos décimas partes del valor límite $(8,10)$.

\section{DISCUSIÓN}

Los resultados obtenidos en relación con la contaminación de los alimentos son preocupantes y a ello se suma el hecho de que la población no tiene un conocimiento real de las características y los problemas asociados con el consumo de estos productos que se ingieren cotidianamente.

Perú es uno de los países que notifican regularmente la superación de límites prohibidos o separados, lo que sitúa a los productos peruanos en mala situación, reduce su credibilidad y la confianza de los consumidores. Pero la situación para el mercado nacional es aún más preocupante, ya que casi nadie controla los productos frescos que ingresan en el mercado local para el consumo. En este sentido, los consumidores están desprotegidos y expuestos a los daños para la salud que ocasiona la ingesta permanente e inadvertida de sustancias tóxicas. Se debe tener en cuenta que en un producto con residuos agroquímicos (tomate, patata, zanahoria, frutas, etc.), incluso si se lava o hierve, las sustancias químicas permanecen y eventualmente causan daño por su acumulación en el organismo (23).

Al haber encontrado arsénico y cadmio, se investigará la presencia de estos metales a partir de 2018 conforme a la regulación europea (14), dado que en el Codex Alimentarius no se establecen límites para ellos (10).
La presencia de antibióticos en la carne es un problema porque su consumo constante por los consumidores peruanos puede generar resistencias antibacterianas (19), lo que pone en riesgo la salud, ya que ante cualquier infección, los antibióticos comunes ya no producen ningún efecto.

Pese a que ninguna muestra de alimentos con arsénico sobrepasa los niveles permitidos, el tema es preocupante, pues, aunque la FDA lo ha retirado de los Estados Unidos de América, es bien sabido que en la cría de pollos se utilizan medicamentos que contienen arsénico para acelerar el crecimiento de las aves (20). Cabe mencionar que ha habido diversos informes internacionales que denuncian la utilización de arsénico en los alimentos, un elemento químico que está estrechamente asociado con cánceres de piel, pulmón y vejiga, por lo que su presencia en los alimentos está muy cuestionada en todo el mundo (21).

Respecto a la importación y exportación de estos productos, los exportadores nacionales son los que están más preocupados por cumplir con los estándares internacionales para llevar sus productos a otros mercados, especialmente los de origen agrícola, puesto que dichos mercados son más exigentes. Si se incumplieran estos estándares, sus productos no se aceptarían. A pesar de ello, los rechazos son frecuentes porque algunos no cumplen con los requisitos sanitarios. La mayoría de los países en el mundo velan por la salud de su población y, por lo tanto, exigen el cumplimiento de las normas internacionales para garantizar que la comida no sea un riesgo para la vida de sus habitantes. A través del Sistema de Alerta Rápida para Alimentos y Piensos (RASFF, por sus siglas en inglés), que opera a nivel internacional, se mantiene una actualización constante de dicha información (22).

Este es el primer estudio que muestra el nivel de contaminación de los alimentos de origen animal y vegetal que fueron monitorizados por la autoridad sanitaria en Perú entre 2011 y 2015. Los informes consultados demuestran que existe una clara deficiencia en los sistemas de procesamiento de alimentos y que, asimismo, hay un alto riesgo de causar daños en la salud de los consumidores.

Según se ha detallado, los controles para el consumo interno no son suficientemente rigurosos en comparación con los realizados para los alimentos destinados a la exportación. Se han encontrado alimentos de consumo masivo que incumplen estas normas por diversos motivos, como, por ejemplo, el tomate por la presencia de pesticidas, la paprika por micotoxinas (ocratoxina A y aflatoxina B1), la carne de cerdo por antibióticos como furazolidona, furaltadona y sus metabolitos, las mandarinas, naranjas y uvas por plaguicidas no autorizados, las mandarinas, espárragos, uvas, plátanos y naranjas por productos químicos no autorizados, y la carne de aves de corral, bovinos, ovinos y caprinos por arsénico.

Esta situación es más grave que cualquiera de los casos de etiquetado falso o engañoso que se hayan notificado recientemente, porque no se trata solamente de un problema de información, sino de falta de inocuidad del producto, de los peligros que supone para la salud de millones de consumidores y de la carencia de protección e información en que se encuentran los consumidores frente a las compras cotidianas que realizan en cualquier mercado del país.

Tras una extensa evaluación y observación de la realidad en el control y supervisión de los productos que se analizan en esta monitorización, se hacen las siguientes recomendaciones:

1. Reorganizar el sistema de control del uso de agroquímicos en el agro a fin de hacerlo más eficiente y resguardar la salud de la población.

2. Declarar dicho control prioridad nacional para implementar programas integrados de manejo de plagas.

3. Establecer metas e indicadores claros para la reducción del uso de plaguicidas en el país.

4. Retirar de manera inmediata los plaguicidas de etiqueta roja del mercado nacional.

5. Promover la producción orgánica como una alternativa para aprovechar los recursos de la agrobiodiversidad.

6. Reformular la estructura y organización del SENASA creando un Directorio Evaluador Científico con representación de sectores de la producción agrícola, agricultores ecológicos, expertos nacionales e internacionales y de los consumidores.

7. Establecer un programa para el "Manejo Integrado de Plagas", fomentar un mayor uso de agentes de control biológico y extractos 
vegetales, reducir la dependencia de plaguicidas químicos y, por lo tanto, desarrollar la agricultura sostenible, así como mejorar la calidad ambiental y de la calidad de vida de los ciudadanos.

8. Las acciones planteadas como respuesta a los problemas descritos deberán planificarse y contar con la

1. Ministerio de Salud del Perú, Dirección General de Salud Ambiental (DIGESA). Ley de Inocuidad de los Alimentos. Decreto Legislativo No. 1062. Lima: Ministerio de Salud del Perú; 2008.

2. Ministerio de Agricultura y Riego del Perú, Servicio Nacional de Sanidad Agraria (SENASA). Reglamento de la Ley de Inocuidad de los Alimentos. Decreto Supremo No. 034-2008-AG. Lima: Ministerio de Agricultura y Riego del Perú; 2008.

3. Ministerio de Salud del Perú, Dirección General de Salud Ambiental (DIGESA). Reglamento de Inocuidad Agroalimentaria. Decreto Supremo No. 04-2011-AG. Lima: Ministerio de Salud del Perú; 2011.

4. Ministerio de Agricultura y Riego del Perú, Servicio Nacional de Sanidad Agraria (SENASA). Plan Anual de Monitoreo de Contaminantes Químicos en Alimentos Agropecuarios Primarios y Piensos de Producción Nacional o Extranjera para el Periodo 2011. Resolución Dictatorial No. 107-2011-AG-SENASA-DIAIA. Lima: Ministerio de Agricultura y Riego del Perú; 2011.

5. Williams-Nguyen J, Sallach JB, BarteltHunt S, Boxall AB, Durso LM, McLain JE, et al. Antibiotics and Antibiotic Resistance in Agroecosystems: State of the Science. J Environ Qual. 2016;45(2):394-406.

6. Aga DS, Lenczewski M, Snow D, Muurinen J, Sallach JB, Wallace JS. Challenges in the Measurement of Antibiotics and in Evaluating Their Impacts in Agroecosystems: A Critical Review. J Environ Qual. 2016;45(2): 407-19.

7. World Health Organization. Antimicrobial Resistance. Global Report on Surveillance. Geneva: WHO; 2014. Disponible en: http: / / apps.who.int/iris/bitstream/ 10665/112642/1/9789241564748_eng.pdf Acceso el 21 de septiembre de 2017.

8. Servicio Nacional de Sanidad Agraria. Informe del Monitoreo de Contaminantes Químicos en Alimentos Agropecuarios Primarios, Año 2011. Lima: SENASA; 2011. Disponible en: http: //www.senasa. gob.pe/senasa /wp-content/uploads / 2016/12/Monitoring-Report-MonitoringQuick-Agropecuarios-Primarios-y-Feedsa\%C3\%B1o-2011 Acceso el 21 de septiembre de 2017.

9. Servicio Nacional de Sanidad Agraria. Informe del Monitoreo de Contaminantes Químicos en Alimentos Agropecuarios Primarios, Año 2012. Lima: SENASA; participación de todos los actores del agrosistema de Perú para lograr introducir los cambios regulatorios que se requieren y disponer de indicadores adecuados para su monitorización y control.

Financiación. El presente estudio no ha sido financiado.

\section{REFERENCIAS}

2012. Disponible en: http://www.senasa. gob.pe/senas a / w p-content/ uploads / 2016/12/-Report-of-WasteMonitoring-Q-\%C3\%Admicro-and-otherContaminants-in-Foods-AgropecuariosPrimarios-a\%C3\%B1o-2012.pdf Acceso el 21 de septiembre de 2017.

10. Servicio Nacional de Sanidad Agraria. Informe del Monitoreo de Contaminantes Químicos en Alimentos Agropecuarios Primarios, Año 2013. Lima: SENASA; 2013. Disponible en: http://www.senasa. gob.pe/senasa/wp-content/uploads / 2016/12/Informe-del-Monitoreo-de-Residuos-Qu\%C3\%ADmicos-y-otros-Contaminantes-en-Alimentos-AgropecuariosPrimarios-a\%C3\%B1o-2013.pdf Acceso el 21 de septiembre de 2017.

11. Servicio Nacional de Sanidad Agraria. Informe del Monitoreo de Contaminantes Químicos en Alimentos Agropecuarios Primarios, Año 2014. Lima: SENASA; 2014. Disponible en: http://www.senasa.gob.pe/ senasa/wp-content/uploads/2016/12/ Informe-del-Monitoreo-de-Residuos-Qu $\%$ C3\%ADmicos-y-otros-Contaminantesen-Alimentos-Agropecuarios-Primarios-a $\%$ C3\%B1o-2014.pdf Acceso el 21 de septiembre de 2017.

12. Servicio Nacional de Sanidad Agraria. Informe del Monitoreo de Contaminantes Químicos en Alimentos Agropecuarios Primarios, Año 2015. Lima: SENASA; 2015. Disponible en: http:/ / www.senasa.gob.pe/ senasa /wp-content/uploads/2016/12/ Informe-del-Monitoreo-Adicional-de-ResiduosQu\%C3\%ADmicos-y-otros-Contaminantesen-Alimentos-Agropecuarios-Primarios-a\%C3\%B1o-2015.pdf Acceso el 21 de septiembre de 2017.

13. Ministerio de Agricultura y Riego del Perú, Servicio Nacional de Sanidad Agraria (SENASA). Productos Agricolas con Informe de Importación Negativa por la FDA del Año 2014 hasta 30.09.2015. Informe No. 0032-2015-MINAGRI-SENASA-DIAIAJCARRASCO. Lima: Ministerio de Agricultura y Riego del Perú; 2015.

14. Comisión Europea. Reglamento (UE) No. 1275/2013: Modificación al Anexo I de la Directiva 2002/32/CE del Parlamento Europeo y del Consejo en lo que respecta a los niveles máximos de arsénico, cadmio, plomo, nitritos, esencia volátil de mostaza e impurezas botánicas perjudiciales. Bruselas: Diario Oficial de la Unión Europea: 2013. Disponible en: https:// www.boe.es / doue/2013/328/L000
Conflictos de intereses. Los autores declaran no tener conflictos de interés

Declaración. Los autores son los únicos responsables de las opiniones expresadas en el manuscrito, que no necesariamente reflejan la opinión o política de la RPSP / PAJPH y / o la OPS.

86-00092.pdf Acceso el 21 de septiembre de 2017.

15. Food and Agriculture Organization. The State of Food and Agriculture 2016. Rome: FAO; 2016. Disponible en: http://www. fao.org/3/a-i6030e.pdf Acceso el 21 de septiembre de 2017.

16. Instituto Nacional de Estadística e Informática. Consumo Per Capita de los Principales Alimentos 2008-2009. Lima: INEI; 2009. Disponible en: https://www. inei.gob.pe/media/MenuRecursivo/publicaciones_digitales/Est/Lib1028/cap01. pdf Acceso el 21 de septiembre de 2017.

17. Ravelo Abreu A, Rubio Armendariz C, Gutierrez Fernandez AJ, Hardisson de la Torre A. Ochratoxin A in foods for human consumption: review. Nutr Hosp. 2012;26(6):1215-26.

18. Escriva L, Font G, Manyes L, Berrada H. Studies on the Presence of Mycotoxins in Biological Samples: An Overview. Toxins. 2017;18;9(8).

19. Landers TF, Cohen B, Wittum TE, Larson EL. A review of antibiotic use in food animals: perspective, policy, and potential. Public Health Rep. 2012;127(1):4-22.

20. Nachman KE, Baron PA, Raber G, Francesconi KA, Navas-Acien A, Love DC. Roxarsone, inorganic arsenic, and other arsenic species in chicken: a USbased market basket sample. Environ Health Persp. 2013;121(7):818-24.

21. Putila JJ, Guo NL. Association of arsenic exposure with lung cancer incidence rates in the United States. PloS one. 2011;6(10):e25886.

22. European Commission, RASFF Food and Feed Safety Alerts. Rapid Alert System for Food and Feed. Brussels: European Commission; 2017. Disponible en: https:// webgate.ec.europa.eu/rasff-window/portal $/$ ? event $=$ notificationsList\&StartRow $=1$ Acceso el 21 de septiembre de 2017.

23. del Puerto Rodríguez AM, Suárez Tamayo S, Palacio Estrada DE. Efectos de los plaguicidas sobre el ambiente y la salud. Rev Cubana Hig Epidemiol. 2014;52(3): 372-87.

Manuscrito recibido el 21 de septiembre de 2017. Aceptado para publicación, tras revisión, el 11 de diciembre de 2017. 
ABSTRACT

Indiscriminate use of pesticides and lack of sanitary control in the domestic market in Peru

Key words Food; pollutants; environmental contamination; government regulation; Peru.
This communication seeks to report the level of contamination of foodstuffs of animal and vegetable origin monitored by a health authority of Peru, namely the National Agrarian Health Service (SENASA), in order to inform consumers and national authorities of the current situation of sanitary control of the Peruvian domestic market.

Information was obtained from reports posted on the SENASA website. The data considered correspond to those present in evaluation reports of all food types of vegetable and animal origin analyzed in the period 2011-2015.

Results for this period show that 202 samples of animal and plant origin were in violation of standards (noncompliant). Overall, $12.68 \%$ of food samples of animal origin and $24.87 \%$ of food samples of vegetable origin were noncompliant. Over the course of the studied period, a $30.73 \%$ increase in the proportion of noncompliant samples was observed, reaching as high as $50 \%$.

The levels of contamination of foods of animal and vegetable origin monitored by SENASA are concerning. It is thus recommended that concrete actions be implemented to address this situation, and that these actions be prioritized and planned with the involvement of all actors in Peru's agricultural system in order to introduce the necessary regulatory changes and establish clear, measurable, and achievable indicators of monitoring and control to protect the health of the population.
RESUMO

\section{Uso indiscriminado de pesticidas e falta de controle sanitário do mercado interno no Peru}

Notificar o grau de contaminação de alimentos de origem animal e vegetal medido pela agência sanitária do Peru (Serviço Nacional de Sanidade Agrária, SENASA) a fim de informar o consumidor e as autoridades nacionais da situação de controle sanitário do mercado interno do país.

A informação foi obtida de informes publicados no website do SENASA. Os dados correspondem aos obtidos de informes de avaliação de todos os tipos de alimentos de origem animal e vegetal analisados no período entre 2011 e 2015.

Os resultados obtidos para o período de estudo revelam que 202 amostras de alimentos de origem animal e vegetal descumprem as normas (não conformidade). O percentual de amostras não conformes foi $12,68 \%$ para os alimentos de origem animal e $24,87 \%$ para os alimentos de origem vegetal. Observou-se neste período aumento de $30,73 \%$ das amostras em não conformidade, inclusive alcançando $50 \%$.

O grau de contaminação dos alimentos de origem animal e vegetal medido pelo SENASA é preocupante. Recomendam-se medidas concretas para enfrentar este problema e tais medidas devem ser priorizadas e planejadas com a participação de todos os interessados diretos do sistema agrícola do Peru a fim de introduzir as mudanças reguladoras necessárias e estabelecer indicadores claros, mensuráveis e alcançáveis para monitoramento e controle para proteger a saúde da população.

Palavras-chave Alimentos; poluentes; poluição ambiental; regulamentação governamental; Peru. 